\title{
The Role of Virtualization in the Small Cell Enabled Mobile Edge Computing Ecosystem
}

\author{
Leonardo Goratti $^{1}$, C.E. Costa ${ }^{1(\bowtie)}$, Jordi Perez-Romero ${ }^{2}$, \\ P.S. Khodashenas ${ }^{3}$, Alan Whitehead ${ }^{4}$, and Ioannis Chochliouros ${ }^{5}$ \\ ${ }^{1}$ CREATE-NET, Via alla Cascata 56/D, Trento, Italy \\ ccosta@fbk.eu \\ 2 Universitat Politecnica de Catalunya (UPC), Barcelona, Spain \\ 3 i2CAT, Barcelona, Spain \\ ${ }^{4}$ IP.Access, Cambourne, UK \\ ${ }^{5}$ Hellenic Telecommunications Organization (OTE), Marousi, Greece
}

\begin{abstract}
Virtualisation is playing a fundamental role in the evolution of telecommunication services and infrastructures, bringing to rethink some of the traditional design paradigms of the mobile network and enabling those functionalities necessary for supporting new complex ecosystems where multiple actors can participate in a dynamic and secure environment. In Small Cell enabled Mobile Edge Computing deployments, the impact of virtualization technologies is significant in two main aspects: the design and deployment of the telecommunication infrastructure, and the delivery of edge services. Besides, the adoption of virtualization technologies has implications also in the implementation of Self Organizing Network (SON) services and in the enforcement of Service Level Agreement (SLA) policies, both critical in the automation of the delivery of multi-tenant oriented services in such complex infrastructure. From the work performed by the H2020 SESAME project, the beneficial use of virtualization techniques emerges in adding network intelligence and services in the network edge. SESAME relays on virtualization for providing Small Cell as a Service (SCaaS) and per operator Edge Computing services, consolidating the emerging multi-tenancy driven design paradigms in communication infrastructures.
\end{abstract}

Keywords: NFV $\cdot$ Small Cell $\cdot$ Virtualization $\cdot 5 \mathrm{G}$

\section{Introduction}

Virtualization technologies decouple software from physical infrastructure, and create virtual resources dedicated to distinct services. This approach fosters an efficient, flexible and dynamically re-configurable usage of the physical resources, and a rapid and efficient service creation process.

While virtualization is a fundamental element of Cloud Computing, it is also making its way in the Telecommunication domain. Through virtualization, network elements, functions and components are released from the limitations of dedicated hardware and physical constraints, allowing the dynamic set up and reconfiguration of services and service providers, and the support the functionalities necessary for implementing multi-tenancy and autonomic management. 
Operators can leverage on virtualization for implementing new business models. An important novelty enabled by multi-tenancy is the possibility of decoupling the Mobile Network Operators (MNO) roles and functions between multiple operators: such as service providers, infrastructure provider and network provider. Besides the MNO, new types of Virtual Network Operators (MVNO) and Over-The-Top (OTT) service providers are enabled to enter in the value chain. For example, Small Cell (SC) operators (SCOs) can leverage on this framework for offering to existing MNOs on-demand access to network resources, acting as a neutral host provider. Venue owners, real estate companies, municipalities, etc., can become SCOs by deploying an appropriate Small Cell access infrastructure and by providing new services, especially in high traffic and with high end-users density delimited places such as office areas, dense urban areas, stadiums, shopping malls, and concert venues [1].

\subsection{SESAME Project}

With the introduction of new paradigms, such as edge computing and radio access (RAN) functional split, telecommunication services and infrastructures are evolving into a new hybrid Cloud-Telco breed of services, where Cloud Computing and telecom infrastructure virtualization are put together for supporting new multi-tenant business models and vertical markets, forcing to rethink some of the traditional design paradigms of the mobile network.

The framework envisaged by the SESAME 5G-PPP EU-funded project [5] supports a hybrid cloud-telecommunication infrastructure, with enhanced multi-tenant cloud services by combining SCs with micro-server facilities at the edge. Its target is to design and develop a novel 5G platform based on Small Cells and Edge Cloud Computing. Featuring a virtualised execution environment and multi-tenancy between network operators, it aims at offering to both network operators and mobile users services that can potentially take advantage of the location of computational resources at the edge of the network.

Various high-impact use cases for Small Cell enabled Edge Cloud Computing [2] and the key stakeholders have been identified. For satisfying their requirements, SESAME innovations focus around three central elements in the wider 5G context:

(i) The placement of network intelligence and applications in the network edge through Network Functions Virtualization (NFV) and Edge Cloud Computing; (ii) the substantial evolution of the Small Cell concept, already mainstream in 4G but expected to deliver its full potential in the challenging high dense 5G scenarios, and; (iii) the consolidation of multi-tenancy in communications infrastructures, allowing several operators/service providers to engage in new sharing models of both access capacity and edge computing capabilities.

The SESAME Small Cell infrastructure integrates, inside the access network infrastructure, a virtualized execution platform (i.e., the Light Data Centre (DC)): a highly manageable clustered edge computing infrastructure that features low-power processors and hardware accelerators for time critical operations. In the Light DC, Virtual Network Functions (VNFs) are deployed, and applications and services executed. 
The SESAME approach allows new stakeholders to dynamically enter the value chain by acting as neutral host providers in high traffic areas where densification of multiple networks is not practical. The optimal management of a CESC deployment is a key challenge of SESAME, for which new orchestration, NFV management, virtualization of management views per tenant, Self-X features and radio access management techniques are developed.

\subsection{Technology Trends in Small Cells}

Some of technology trends that can be identified for the Small Cells are enabled by virtualization capabilities of the infrastructure:

Functional Split in Small Cells. The functional split defines which of the functions typically run in the eNodeB can be executed in the cloud. The Small Cell Forum (SCF) has recognized multiple functional splits of the Small Cell networking layers for different use cases $[3,4]$. At which point of the protocol stack perform the functional split, together with the decision of where to execute virtual the network functions with respect to physical small cell functions, are two crucial aspects in Small Cell virtualization that influences the flexibility and the management of RAN optimization.

Multitenancy Capabilities. Traditional mobile operators deploy their own network infrastructure in competition with others. In multi-tenancy approaches, instead, infrastructure and resources are shared between multiple operators, encouraging a more dynamic and scalable market: operators can focus on their core business and differentiate their offer based rather delegating network connectivity maintenance to a third party. SESAME approach allow several operators/service providers to engage in new sharing models, obtaining higher capacity on the access side and exploiting edge computing capabilities.

Radio Access Network (RAN) Sharing. Multi-tenancy poses unprecedented challenges to the owner of the shared RAN in relation to network planning. The management of tenants may need to handle drastic variations in the network's traffic demand, e.g. due to the aggregation of new tenants. Moreover, the new aggregated traffic is tenant-specific, meaning that some characteristics (e.g. busy hour, type of services, etc.) may substantially differ from tenant to tenant, particularly if these tenants correspond to different vertical sectors. For these reasons, flexibility is crucial for an efficient and cost-effective multi-tenancy implementation. There are several approaches for RAN slicing in relation to different aspects such as the degree of isolation that can be achieved between tenants or the degree of customization, which impacts on the capability to customize RRM/Self-X functions on a per tenant basis.

Cloud-Enabled Small Cell. Making computing capabilities available at the network edge allows providers to locate services nearer to the mobile subscribers, thus enabling accelerated services, content and application. SESAME approach proposes to deploy a these capabilities between the mobile core and the Radio Access Network (RAN). Typical services which can benefit from mobile-edge computing include Internet-of-Things, augmented reality and data caching. 
Network Slicing. The SC network is partitioned in multiple isolated slices: Virtualised resources and small cells capacity is partitioned into slices configured on the requirements of multiple vertical markets.

Self-Organising Network (or Self-X Functions). The complexity of the highly dense environments where Small Cells are envisaged to be deployed require the introduction mechanisms for reducing or even removing the need for manual network optimization tasks, thus enabling autonomic capabilities to the network. Through the implementation of SON features the network framework is are able to dynamically tune global operational settings of the SC (e.g., transmit power, channel bandwidth, electrical antenna tilt) as well as specific parameters corresponding to Radio Resource Management (RRM) functions (e.g., admission control threshold, handover offsets, packet scheduling weights, etc.). The development of Self-X functions benefits from the availability of a virtualized execution platform provided at the RAN and by the use of NFV and MEC technologies. The introduction of SON architectures have a positive impact in reducing the network operating costs by minimizing human errors [6].

\section{The Role of Virtualization}

Virtualization is fundamental for an effective and exploitable deployment of computation capabilities at the mobile network edge, allowing managing and orchestrating network services from different services providers in a dense small cell scenarios and different use cases.

Cloud computing approaches, such as Infrastructure-as-a-Service (IaaS), can be adopted in order to facilitate the decoupling between providers and their roles: i.e. the service provider, the infrastructure provider and the network provider.

To achieve a mapping of the conventional telecommunication services to the new Cloud-enabled infrastructures, Service Function Chaining techniques can be applied. Software Defined Networking (SDN) mechanisms for Service Function Chaining (SFC) are the most prominent candidates to enforce traffic steering through a logical network graph and to achieve certain service functionality among the virtualized components. Extending the concept of SFC in the context of the 5G ecosystem requires deeper understanding on the NFV concepts in such a scenario. Carefully identifying the requirements of the specific setup is important in order to choose the most suitable mechanisms and protocols to establish the desired functionality.

From the standardization point of view, the ETSI NFV Industry Specification Group (ISG) has developed the Management and Orchestration (MANO) framework, a reference for managing virtualised environments [7].

\subsection{Virtualisation of the Communication Infrastructure}

Virtualisation of the communication infrastructure, such as core/edge network elements and access points/macrocells, finds its application in Small Cells and Small Cell-as-aService (SCaaS) scenarios. If we consider mobile networks, there exist several virtualized deployments of the common LTE functional blocks, such as EPC, BSS, HSS, RAN, etc. 
The management of dense SC networks includes mitigating interference and assigning resources dynamically. SC installation is an effective way to achieve greater performance and capacity to both indoor and outdoor places but this brings complexity. In order to share SC networks between multiple operators, it is necessary to logical partitioning of the SC network into multiple isolated slices. Virtualizing part of RAN functionalities allow partitioning small cells capacity between multiple tenants. Small Cell functions virtualization gives improved ability and agility to manage dense SC networks, e.g. mitigating interference and assign resources dynamically.

SESAME relays on virtualization to develop a SC operating model under which multi-operators (i.e., multi-tenancy) can coexist.

\subsection{Virtualization of Edge Services}

Virtualization of edge services offers computing capabilities at the network edge and brings different services near to the mobile subscribers such as augmented reality (AR), caching, security services, multimedia services, big data. In such a scenario, operators can differentiate their offer based on services rather than on network connectivity.

Network Functions (NFs) - such as caching proxies, firewalls, load balancers or intrusion detection systems (IDSs) - traditionally deployed as middleboxes (running in dedicated hardware), can be virtualized and linked together e.g. using SDN based approaches.

SESAME provides enhanced multi-tenant edge cloud services combining SCs with micro-server facilities, supporting virtualised execution environment. SESAME leverages on the capability to deliver intelligence directly to the network's edge, in the form of virtual network appliances.

Using Service Function Chaining (SFC) capabilities, it is possible to create a new complex service defining an ordered list of a network services (e.g. firewalls, load balancers) that are connected together in the network.

\section{Conclusions}

Virtualization plays a fundamental role in the new hybrid Cloud-Telco infrastructure, such as the Edge Clout Computing Small Cell architecture proposed by SESAME [8], and forces to rethink some of the traditional design paradigms of the mobile network. In particular, virtualization has a fundamental role in:

- Supporting an infrastructure that is sustainable and reconfigurable, both from the SC RAN and Services point of view;

- Making the SC infrastructure and resources shared between operators, with a transparent and neutral approach;

- Accelerating the creation of innovative services with superior quality of experience through mobile edge computing;

- Enabling Self-X functionalities, capable of optimise the usage of radio, network, storage and computing resources. 
Acknowledgments. This work has been supported by the EU funded H2020 5G-PPP project SESAME under the grant agreement no. 671596

\section{References}

1. Small Cell Forum (SCF): Small Cell Virtualization: Functional Splits and Use Cases - SFC 159.06.02 (2016)

2. Belesioti, M., Chochliouros, I. (eds.): System Use Cases and Requirements, Deliverable D2.1 of the SESAME project, December 2015

3. Small Cell Forum (SCF): Virtualization in Small Cell Networks - SCF 154.07.02 (2015)

4. Small Cell Forum (SCF): Business case elements for small cell virtualisation - SCF 158.05.1.04 (2015)

5. Small cEllS coordinAtion for Multi-tenancy and Edge services (SESAME) 5G-PPP project. http://www.sesame-h2020-5g-ppp.eu/

6. Ramiro, J., Hamied, K.: Self-Organizing Networks: Self-Planning, Self-Optimization and Self-Healing for GSM. UMTS and LTE. Wiley, Hoboken (2012)

7. European Telecommunications Standards Institute (ETSI): ETSI GS NFV-MAN 001 (V1.1.1): Network Function Virtualisation (NFV); Management and Orchestration. ETSI, Sophia-Antipolis (2014)

8. Giannoulakis, I. (ed.): SESAME Final Architecture and PoC Assessment KPIs, Deliverable D2.5 of SESAME project, December 2016 